\title{
Von der Häufigkeit seltener Erkrankungen ...
}

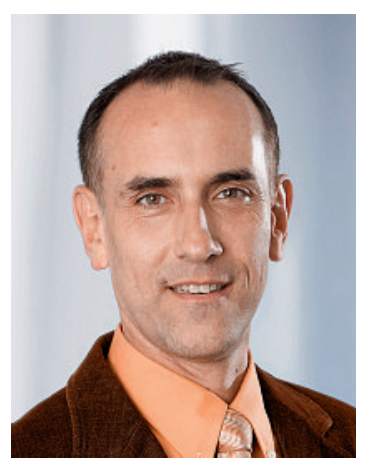

Krankheiten niedriger Prävalenz bezeichnet man als Orphan diseases bzw. seltene Erkrankungen. In Europa spricht man von einer seltenen Erkrankung, wenn weniger als eine Person auf 2000 Einwohner betroffen ist. Von den weltweit etwa 30000 bekannten Krankheiten fallen 20 bis 25 Prozent, also rund 6000 bis 7500 Krankheiten, unter die Rubrik «selten»; etwa vier Fünftel dieser Erkrankungen sind genetisch determiniert.

Extrapoliert man diese Zahlen nun auf Europa, sind seltene Erkrankungen keineswegs selten: absolut betroffen sind rechnerisch zwar «nur» weniger als 250000 Patienten, potentiell reden wir jedoch von mehr als der Bevölkerung Skandinaviens zusammen, nämlich von über 25 Millionen (!) Betroffenen. Etwas weniger dramatisch scheint die Situation in der Schweiz zu sein: bei knapp acht Millionen Einwohnern resultiert eine Absolutzahl von 4000 Patienten; aber die Realität zeigt auch hier, dass über 6 Prozent der Schweizer Bevölkerung zu den potentiell Betroffenen gehört und damit knapp 500000 Menschen!

\section{Seltene Krankheiten tangieren auch die Gesellschaft und damit nicht zuletzt die Frage der Finanzierung.}

Neben dem Kriterium der Anzahl Betroffener beinhaltet der Begriff Orphan diseases in der Medizin von heute, wo wir allenthalben mit EBM (Evidence Based Medicine) konfrontiert werden, zusätzlich etwas Aussergewöhnliches, etwas Problematisches: nämlich tiefe Fallzahlen, fehlende Erfahrung, unsystematische Erfassung, kaum vorhandene Perzeption.

Mit der rasanten Entwicklung der molekularen Diagnostik und der massenspektrometrischen Analytik stehen neue und sichere(re) Diagnostikmöglichkeiten für diese bis anhin verwaisten Erkrankungen zur Verfügung. So lässt sich ein Verdacht klarer bestätigen und lassen sich vielleicht in absehbarer Zeit sogar kostengünstige Screenings wie z.B. ehedem PKU (Phenylketonurie) realisieren. Der Vorteil des molekulargenetischen Ansatzes liegt in der kausalorientierten und spezifischen Zuordnung der Erkrankung. Hiermit könnte sich ein Fenster öffnen, das exakte Einblicke in die Variabilität der verschiedenen Gruppen seltener Erkrankungen und neue, womöglich individualisierte Therapieansätze liefert.
Angesprochen ist auch die Pharma-Industrie: Bedingt durch geringe Prävalenz der einzelnen Erkrankung und entsprechend reduzierte Absatzmärkte resultiert ein entsprechend marginales oder zumindest zurückhaltendes Interesse es wäre wünschenswert, dass sich mehr Unternehmen mit speziellen Forschungs- und Entwicklungsprojekten für Patienten mit seltenen Erkrankungen engagieren.

\section{Betroffene brauchen unsere Solidarität und das Engagement der Industrie und} Politik.

Neben den diagnostischen und therapeutischen Aspekten tangieren seltene Krankheiten jedoch auch die Gesellschaft und damit die Frage der Finanzierung.

Kürzlich fand hierzulande die Diskussion anlässlich eines Bundesgerichtsurteils zu dieser Thematik statt. Für das Gericht stand fest, dass nicht alles, was medizinisch machbar sei, auch durch die Grundversicherung bezahlt werden müsse. Das Bundesgericht setzte mit Verweis auf die gesundheitsökonomische Literatur ein Kostendach von maximal ca. 100000 Franken pro gerettetes Lebensjahr an.

Die Diagnostik und Therapie seltener Erkrankungen ist kostenintensiv. Damit wir Patienten mit seltenen Krankheiten behandeln können, ist

1. eine entsprechende Sensibilisierung der Öffentlichkeit gefragt,

2. ein umfassender Einsatz mit Forschungsanstrengungen der Wissenschaftler vonnöten,

3. die Unterstützung der Politik unabdingbar,

4. die Hilfe der Industrie essentiell,

5. eine gute Planung von Förderinstrumenten wichtig und 6. vor allem eine gute Vernetzung der Betroffenen in Selbsthilfeorganisationen notwendig.

Dieser Mehrpunkteplan sollte die Forschung voranbringen, um neue, wirksame und im Idealfall kostengünstige Therapien zu entwickeln.

Erste Schritte in der Schweiz sind erfolgt: Die Gründung der Interessengemeinschaft IG Seltene Krankheiten unter Mitbeteiligung der FMH steht bevor. Sie gibt Betroffenen eine Stimme und vertritt deren Anliegen gegenüber der Industrie, Politik und Öffentlichkeit. Zudem erarbeitet das Bundesamt für Gesundheit zurzeit ein Konzept zum Umgang mit seltenen Krankheiten. Denn deutlich mehr Patientinnen und Patienten als vermutet leiden an einer seltenen Erkrankung - auch sie sollen auf die Solidarität der Gesellschaft zählen können.

Dr. med. Gert Printzen, Mitglied des Zentralvorstandes, Verantwortlicher Ressort Heilmittel 